\title{
SSEA4: a marker leading the way towards curing diabetes?
}

"The quest for adult stem cells in the pancreas has raised a lot of expectations, but also a lot of controversy."

\section{KEYWORDS: $\beta$-cell regeneration $\approx$ islet stem cells $\approx$ pancreatic stem cells}

\section{Stem cells \& diabetes}

Type 1 diabetes, also known as juvenile-onset diabetes, is mostly diagnosed in children and young adults. Life-long insulin treatment is required, but does not cure the disease. Transplantation of pancreatic insulin-producing $\beta$ cells from organ donors represents a curative treatment. However, it is hampered by the shortage in donor organs from which the $\beta$ cells can be isolated, and by the need of continuous immune suppression. For years, researchers have tried to solve the first problem, namely by searching for alternative sources of transplantable $\beta$ cells or surrogate $\beta$ cells. Another appealing possibility would be to stimulate regeneration of the $\beta$ cells in the patient's pancreas. Increasing the number of $\beta$ cells could also be beneficial in patients suffering from Type 2 diabetes, namely when their $\beta$ cell number has become insufficient to cope with the body's increasing demand for insulin. For regenerative or cell-replacement therapies, it would be significant if the pancreas contains a population of stem cells. Pancreatic stem cells could either be isolated and used to generate more $\beta$-cell progeny in culture for use as a graft, or alternatively they could be stimulated with drugs to regenerate or expand the $\beta$-cell mass within the pancreas.

"SSEA4 is a recognized marker of pluripotent stem cells, such as embryonic stem cells, and not of multipotent stem cells."

In the last decade, we have witnessed a stem cell 'hype' with many researchers trying to identify and isolate stem cells in a variety of tissues or organs. The quest for adult stem cells in the pancreas has raised a lot of expectations, but also a lot of controversy. At present, there is no generally accepted proof for their existence. The idea that the postnatal pancreas still retains undifferentiated progenitor cells 'leftover' from embryonic/fetal development, has been challenged and it may be that the pancreas differs, in this respect, from other organs [1]. It should be noted that there are also alternative possibilities to generate new $\beta$ cells, such as the transdifferentiation or reprogramming of already differentiated exocrine, non- $\beta$ endocrine or other cell types $[2,3]$. Another possibility is the generation of $\beta$ cells from pluripotent stem cells, such as embryonic stem cells [4].

\section{SSEA4 as a prospective pancreatic stem cell marker}

It would be beneficial to our understanding of the role that stem cells may play in pancreatic tissue regeneration to find reliable adult stem cell biomarkers. Furthermore, biomarkers expressed on the stem cell surface would greatly facilitate isolation of adult stem cell populations in order to examine and exploit their regenerative potential. The 'hypothesis article' reported by Alberto Hayek's group in San Diego raises new hope that stem cells could be identified in the adult human pancreas [5]. They presented evidence that SSEA4 may represent a marker for multipotent stem cells in the pancreas. As this antigen is expressed on the cell surface, it can be used to isolate the putative stem cells by cytometry with specific antibodies. SSEA4 is a recognized marker of pluripotent stem cells, such as embryonic stem cells, and not of multipotent stem cells. Actually, SSEA4 expression is lost from stem cells as they start to differentiate. It should, therefore, not be expected on multipotent stem cells such as those that are sought in the pancreas, although the authors point to two previous studies where it was found on multipotent stem cells in the embryonic liver and brain [5].

What is the evidence in this study that SSEA4 represents a marker for pancreatic stem cells? First, they dissociated adult human pancreas' into single cells and separated SSEA $4^{+}$and

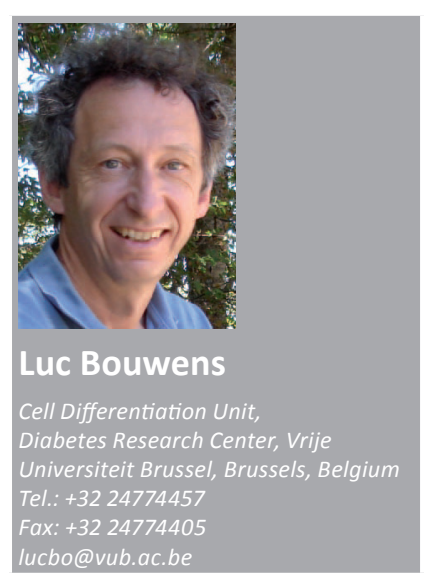

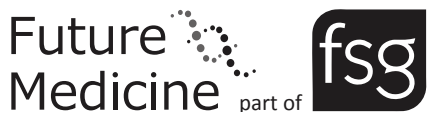


SSEA4 cells by flow cytometry. They compared the expression of certain mRNAs by quantitative reverse transcriptase PCR and found a higher relative expression of OCT4, a marker of pluripotency, as well as c-MET, CD133 and SOX9, which have been previously reported to represent pancreatic progenitor markers (see below). This might fit with the expected phenotype of putative pancreatic stem cells; however, there is a caveat since these markers are also more broadly expressed in differentiated pancreatic cells. C-Met, the receptor for HGF, is also expressed, for example, on $\beta$ cells, and CD133 and SOX9 in the exocrine duct cells [6-8]. The SSEA4 ${ }^{+}$cells represented $17-30 \%$ of all cells, which corresponds to the percentage of duct cells in human pancreas tissue [9].

\section{“...final proof for the stemness of the} reported pancreatic SSEA4 cells can only be given by clonal analysis."

A second major argument is that in fetal human pancreas, SSEA4 was found to colocalize with Ngn3. Although in the embryonic pancreas Ngn3 is not a marker of the multipotent pancreatic progenitor cells, it is expressed by the progenitor cells of all endocrine islet cell types. Therefore, this observation represents a valuable argument. Furthermore, the authors tested the differentiation potential of sorted SSEA4 ${ }^{+}$and SSEA4 cells in a special culture medium and found that expression of Ngn3 mRNA increased during culture of the SSEA4 ${ }^{+}$cells. However, they have to concede that the overall expression of Ngn3 mRNA was low. Expression of this transcription factor is generally considered as evidence of an islet progenitor phenotype, but the significance of low-level expression of $\mathrm{Ngn} 3$ has been put in question, since a low level of expression is also observed in fully differentiated islet cells [10]. After several days culture in the presence of high glucose concentration and several other supplements, an increased expression was observed at the mRNA level for the transcription factors Pdx1 and Nkx6.1, and for the hormones insulin, glucagon and somatostatin. The increase was mainly seen in the SSEA4 ${ }^{+}$ cells and to a lesser extent in the SSEA4- cells. This observation is taken as evidence for differentiation of SSEA4 ${ }^{+}$stem/progenitor cells into endocrine cell types. Another explanation could be that some cells in the SSEA4 ${ }^{+}$fraction were pre-existing endocrine cells and that they increased the transcription of the mentioned genes in the specific culture conditions to which they were applied. This possibility is supported by the observation that some SSEA4 ${ }^{+}$ cells coexpressed insulin in adult pancreas tissue. It is a well-known phenomenon that $\beta$ cells can degranulate or even dedifferentiate following their isolation from the pancreas and that these cells can eventually re-express their original phenotype later on when the culture conditions are appropriate [1]. Dedifferentiated $\beta$ cells can express stem cell markers such as Ngn3, Oct4 and Nanog [12].

As with other putative stem cell markers that have been previously reported, a possible confounding problem is that the marker in question is expressed by several cell types. According to the present study, SSEA4 is expressed by some of the exocrine acinar cells, duct cells and islet cells. As the authors of this paper rightly conclude, final proof for the stemness of the reported pancreatic SSEA4 ${ }^{+}$cells can only be given by clonal analysis. Subsequently it must be demonstrated that single SSEA4 ${ }^{+}$cells are able to self-renew and give rise to clonal colonies and that cells in these colonies can differentiate to pancreatic hormone-expressing cells. Stem cells endowed with the capacity to form multicellular, multilineage 'organoids' starting from a single cell in vitro have been demonstrated, such as in the small intestine and colon [13].

\section{Other pancreatic stem cell markers}

Other presumptive markers to prospectively identify and isolate stem cells from pancreas have been reported along with clonal and/or multipotential capacity in cells from adult mouse pancreas. These were identified by expression of ALDH1 [14], CD133 coexpressed with c-Met [15] or CD133 coexpressed with PDGRa (receptor for PDGF) [16]. It is not yet clear:

- Whether these findings are reproducible in other research groups;

- What the relationship is between progenitor cells reported in the different studies or whether several subpopulations exist;

- Whether such stem/progenitor cells also reside in human pancreas.

\section{Perspective}

Before these discoveries can be translated into clinical applications such as cell transplantation, it must be demonstrated that when hormoneexpressing cells are derived, they are functional and can appropriately secrete insulin in response to glucose similar to normal $\beta$ cells do. 
In addition, in the case of Type- 1 diabetes, there is still the problem of recurrent autoimmunity against the regenerated or transplanted $\beta$ cells that remains to be solved. In the case of transplantation, allograft rejection still represents a major challenge. Also, we have to wonder what may be the risks of transplanting cells that may not be fully differentiated, or of administering growth factors to activate stem cells in the human body. Clearly, there is still a long way to go.

\section{References}

1 Stanger BZ, Tanaka AJ, Melton DA. Organ size is limited by the number of embryonic progenitor cells in the pancreas but not the liver. Nature 445(7130), 886-891 (2010).

2 Baeyens L, Bonné S, Bos T et al. Notch signaling as gatekeeper of rat acinar-to-betacell conversion in vitro. Gastroenterology 136(5), 1750-1760 (2009).

3 Desgraz R, Bonal C, Herrera PL. $\beta$-cell regeneration: the pancreatic intrinsic faculty. Trends Endocrinol. Metab. 22(1), 34-43 (2011).

4 Mfopou JK, Chen B, Sui L, Sermon K, Bouwens L. Recent advances and prospects in the differentiation of pancreatic cells from human embryonic stem cells. Diabetes 59(9), 2094-2101 (2010).

5 Afrikanova I, Kayali A, Lopez A, Hayek A. Is stage-specific embryonic antigen 4 a marker for human ductal stem/progenitor cells? BioResearch 1(4), 184-119 (2012).

6 Demirci C, Ernst S, Alvarez-Perez JC et al. Loss of HGF/c-Met signaling in pancreatic $\beta$-cells leads to incomplete maternal $\beta$-cell

\section{Financial \& competing interests disclosure}

The author has no relevant affliations or financial involvement with any organization or entity with a financial interest in or financial conflict with the subject matter or materials discussed in the manuscript. This includes employment, consultancies, honoraria, stock ownership or options, expert testimony, grants or patents received or pending, or royalties.

No writing assistance was utilized in the production of this manuscript.

adaptation and gestational diabetes mellitus. Diabetes 61(5), 1143-1152 (2012).

7 Lardon J, Corbeil D, Huttner WB, Ling Z, Bouwens L. Stem cell marker prominin-1/ AC133 is expressed in duct cells of the adult human pancreas. Pancreas 36(1), e1-e6 (2008).

8 Kopp JL, Dubois CL, Schaffer AE et al. Sox $9^{+}$ ductal cells are multipotent progenitors throughout development but do not produce new endocrine cells in the normal or injured adult pancreas. Development 138(4), 653-665 (2011).

9 Bouwens L, Pipeleers DG. Extra-insular beta cells associated with ductules are frequent in adult human pancreas. Diabetologia 41(6), 629-633 (1998).

10 Wang S, Jensen JN, Seymour PA et al. Sustained Neurog 3 expression in hormoneexpressing islet cells is required for endocrine maturation and function. Proc. Natl Acad. Sci. USA 106(24), 9715-9720 (2009).

11 Bar Y, Russ HA, Sintov E, Anker-Kitai L, Knoller S, Efrat S. Redifferentiation of expanded human pancreatic $\beta$-cellderived cells by inhibition of the
NOTCH pathway. J. Biol. Chem. 287(21), 17269-17280 (2012).

12 Talchai C, Xuan S, Lin HV, Sussel L, Accili D. Pancreatic beta cell dedifferentiation as a mechanism of diabetic beta cell failure. Cell 150, 1223-1234 (2012).

13 Barker N, Bartfeld S, Clevers H. Tissueresident adult stem cell populations of rapidly self-renewing organs. Cell Stem Cell 7(6), 656-670 (2010).

14 Rovira M, Scott SG, Liss AS, Jensen J, Thayer SP, Leach SD. Isolation and characterization of centroacinar/terminal ductal progenitor cells in adult mouse pancreas. Proc. Natl Acad. Sci. USA 107(1), 75-80 (2010).

15 Oshima Y, Suzuki A, Kawashimo K, Ishikawa $\mathrm{M}$, Ohkohchi N, Taniguchi H. Isolation of mouse pancreatic ductal progenitor cells expressing CD133 and c-Met by flow cytometric cell sorting. Gastroenterology 132(2), 720-732 (2007).

16 Hori Y, Fukumoto M, Kuroda Y. Enrichment of putative pancreatic progenitor cells from mice by sorting for prominin 1 (CD133) and platelet-derived growth factor receptor beta. Stem Cells 26(11), 2912-2920 (2008). 